\title{
Deep sequencing reveals an association between HIV-1 subtype $C$ mutations in gp41 MPER epitopes and mother-to-child transmission
}

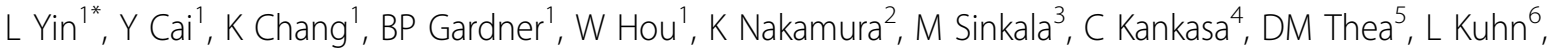 \\ GM Aldrovandi', MM Goodenow ${ }^{1}$
}

From AIDS Vaccine 2012

Boston, MA, USA. 9-12 September 2012

\section{Background}

Enhanced HIV-1 mother-to-child transmission (MTCT) by high maternal anti-gp41 antibody titer led to the hypothesis that transmitting mothers would have greater diversity in membrane-proximal external region (MPER) and mutated amino acid residues associated with resistance to gp41 antibodies.

\section{Methods}

Pyrosequences of HIV-1 subtype-C gp41 heptad repeatregion 2 (HR2), MPER, and membrane-spanning domain (MSD) were generated from 2,000 plasma viral copies/subject from four mothers who transmitted via breast feeding (TM) and four non-transmitting mothers (NTM) in a matched case control study. A bioinformatic pipeline with rigorous quality controls generated $\sim 50,000$ quality pyrosequences/subject and provided 25 -fold coverage of input virus populations. Population genetic algorithms clustered pyrosequences at $3 \%$ genetic distance to study biodiversity using rarefaction/Chao1. Frequency distribution of cluster sizes defined population structure. Consensus sequences constructed from bioclusters for each subject were aligned to an HIV-1 subtype-C consensus sequence to determine number and frequency of nonsynonymous substitutions at each position and to identify mutations by HIV Molecular Immunology Database. Groups were compared using paired t-test.

\section{Results}

Sequences in MPER were more polymorphic than in HR2 or in MSD. TM had more diverse MPERs than
NTM $(p=0.02)$. The number of clusters calculated from rarefaction curves was $62( \pm 35)$ for $\mathrm{TM}$ vs $35( \pm 28)$ for NTM. The Chao1-estimated maximum number of variants within populations was $106( \pm 51)$ for TM vs 59 $( \pm 65)$ for NT. Low fit viruses ( $\leq 5$ sequences/biocluster) contributed to differences in biodiversity between TM $[71.1( \pm 9.7) \%]$ and NTM $[63.5( \pm 14.2) \%]$. Polymorphisms at residues within 4E10 (W672V, F673L, D674S, T676I, and W680G) and 2F5 (D664S) were confined exclusively to viruses from TM mothers. Viral variants with positively charged hydrophilic MPER occurred more frequently in TM than in NTM.

\section{Conclusion}

HIV-1 subtype-C variants with high biodiversity correlated with polymorphisms in MPER and were associated with MTCT, which may reflect increased immune selection and have implications for vaccine design.

\section{Author details \\ ${ }^{1}$ University of Florida, Gainesville, FL, USA. ${ }^{2}$ Childrens Hospital of Los Angeles, Los Angeles, CA, USA. ${ }^{3}$ Lusaka District Management Team, Lusaka, Zambia. \\ ${ }^{4}$ University Teaching Hospital, University of Zambia, Lusaka, Zambia. ${ }^{5}$ Boston University School of Public Health, Boston, MA, USA. ${ }^{6}$ Columbia University, New York, NY, USA. ${ }^{7}$ Childrens Hospital of Los Angeles, Los Angeles, CA,} USA.

Published: 13 September 2012

doi:10.1186/1742-4690-9-S2-P154

Cite this article as: Yin et al.: Deep sequencing reveals an association between HIV-1 subtype $C$ mutations in gp41 MPER epitopes and mother-to-child transmission. Retrovirology 2012 9(Suppl 2):P154.

"University of Florida, Gainesville, FL, USA

Full list of author information is available at the end of the article

(c) 2012 Yin et al; licensee BioMed Central Ltd. This is an Open Access article distributed under the terms of the Creative Commons 